Submission ID: 43979

\title{
Can the Information on the Shale Reservoirs Mineral Composition be
} Useful for Petrophysicists?

B.N. Enikeev* (Pangea Inc.)

\section{SUMMARY}

The report, based on the processing of several data sets on shale reservoirs, provides conclusions on the possibility and specificity of synthesizing multidimensional petrophysical interrelations between the mineral composition of the rock and the quantitative content of organic substance in it. For this purpose, author employs regression and cluster analysis. The limitations, adaptability and accuracy of this approach are discussed in comparison with known pair relationships. The use of such models for clay reservoirs is to increase the accuracy of petrophysical modeling and petrophysical inversion. 


\section{Может ли быть полезной для петрофизиков информация о компонентном составе сланцевых коллекторов?}

Б.Н. Еникеев (АО “ПАНГЕЯ”)

Введение

Практика публикаций корреляционных полей на тернарных диаграммах, показывающая относительное расположение вычленяемых фаций сланцевых коллекторов или компонент породы широко распространена во всём мире. Однако практика анализа многомерных статистических взаимосвязей компонентного см состава с другими свойствами мало распространена.

Простые алгоритмы выделение перспективных интервалов по данным метода естественной радиоактивности (W.Fertl) или акустического каротажа и метода сопротивления (Q.R.Passey) подобную информацию не учитывают. Вместе с тем игнорирование в различии содержания компонент для которых характерные разные плотности и скорости распространения упругих волн представляется легкомысленным.

С формально-статистической точки зрения одна из основных трудностей интерпретации сложных коллекторов - повышенное число минеральных компонент, вклад которых которые нельзя игнорировать и при этом слабая корреляция содержания разных компонент друг с другом. Более того, обычно не используется информация и об областях с крайне редко встречающимся сочетанием компонент. В этой связи особый интерес (как для задачи петрофизического моделирования разреза, так и для решения обратных задач) представляет изучения взаимосвязей содержания компонент друг с другом [1-3].

В отечественных работах учет содержания минеральных компонент встречается редко и неполно. В публикациях исследователей других стран подобная информация также приводится только в ограниченном объёме. В докладе описывается сбор и исследование информации о наличии взаимосвязи ТОС и содержания минеральных компонент породы.

\section{Организация данных}

Организация поиска public domain данных и их интеграции из сегментов во многом предопределяет получаемый результат, его устойчивость и практическую ценность. Значимая неоднозначность выделения минеральных компонент и сложность проведения измерений приводит к сравнительно редкой встречаемости желаемой информации. Кроме того различие в практике процедур селекции образцов, методик подготовки образцов к исследованиям и самих измерений, не говоря уже о различии процедур осадконакопления и постседиментационной эволюции, вносит заметную рассеивающую компоненту

Тем не менее, автор доклада счел возможным опираться на публикации в известных и доступных ему научных журналах и на данные диссертаций, размещенных в открытом доступе. В итоге предварительного просмотра значительного публикаций было принято решение в первую очередь собирать и использовать данные, в которых помимо содержания карбонатной, полевошпатовой и глинистой компоненты имеется информация о содержании пирита, TOC, Rо и глубине залегания пород. Массив с содержанием всех указанных атрибутов мы далее называем базовым.

К сожалению, оказалось, что данные о коллекторских свойствах, площади поверхности и плотности (не говоря уже про их мощность) встречаются существенно реже. Другим и возможно более объективным направлением является сбор информации о содержании химических элементов, в том числе индикаторов зон окисления и восстановления. Ограниченность объёма публикации не позволяет поместить здесь все использованные литературные ссылки.

В итоге работы нами были сформированы таблицы по данным из Северной Дакоты, а также по смешанным данным по объектам из США и Канады и данные по объектам из Китая. 


\begin{tabular}{|l|l|l|c|}
\hline № & \multicolumn{1}{|c|}{ Отложения и источники } & \multicolumn{1}{|c|}{$\begin{array}{c}\text { Отличие массива от } \\
\text { базового }\end{array}$} & $\begin{array}{c}\text { Максимальное число строк в } \\
\text { массиве данных }\end{array}$ \\
\hline 1 & $\begin{array}{c}\text { Williston, Bakken } \\
\text { Северная Дакота [4] }\end{array}$ & $\begin{array}{l}\text { Только ТОС, S1, S2,S3, Ro и } \\
\text { карбонатность }\end{array}$ & 685 \\
\hline 2 & $\begin{array}{l}\text { Midland Basin Wolfcamp/ } \\
\text { Cline Shale [5] }\end{array}$ & $\begin{array}{l}\text { Есть дополнительно } \\
\text { плотность и более детальная } \\
\text { минералогия }\end{array}$ & 65 \\
\hline 3 & $\begin{array}{l}\text { Сланцевые коллектора } \\
\text { Китая (из 19 публикаций) }\end{array}$ & $\begin{array}{l}\text { Интервал глубин от 20 до } \\
\text { 5500, возраст до Кембрия }\end{array}$ & 322 \\
\hline
\end{tabular}

\section{Известные исторические взаимосвязи и используемое программное обеспечение}

Исторически помимо тернарных диаграмм исследователями отмечен целый ряд парных взаимосвязей в декартовых координатах. Широкую популярность такие взаимосвязи не приобрели. Вместе с тем, уместно упомянуть о наличии линейных и гиперболических связей ТОС с карбонатностью (для отложений с преобладанием карбонатного материала), а также с содержанием кварца и пирита. При обработке нами использовался пакет ориентированный на решение этих задач, включающий средства визуализации, построения регрессий (методом выбора оптимального подмножества регрессоров методом ЭфроимсонаМиллера и методы кластерного анализа (методы средних и смесей распределений).

\section{Обработка данных по массивам данных}

Остановимся подробнее на полученных результатах. Первый массив был взят исключительно из-за его размера и доступности и возможности проверить на нем наличие хотя бы намека на какую-то закономерность. Результаты сведены на Рис 1 .

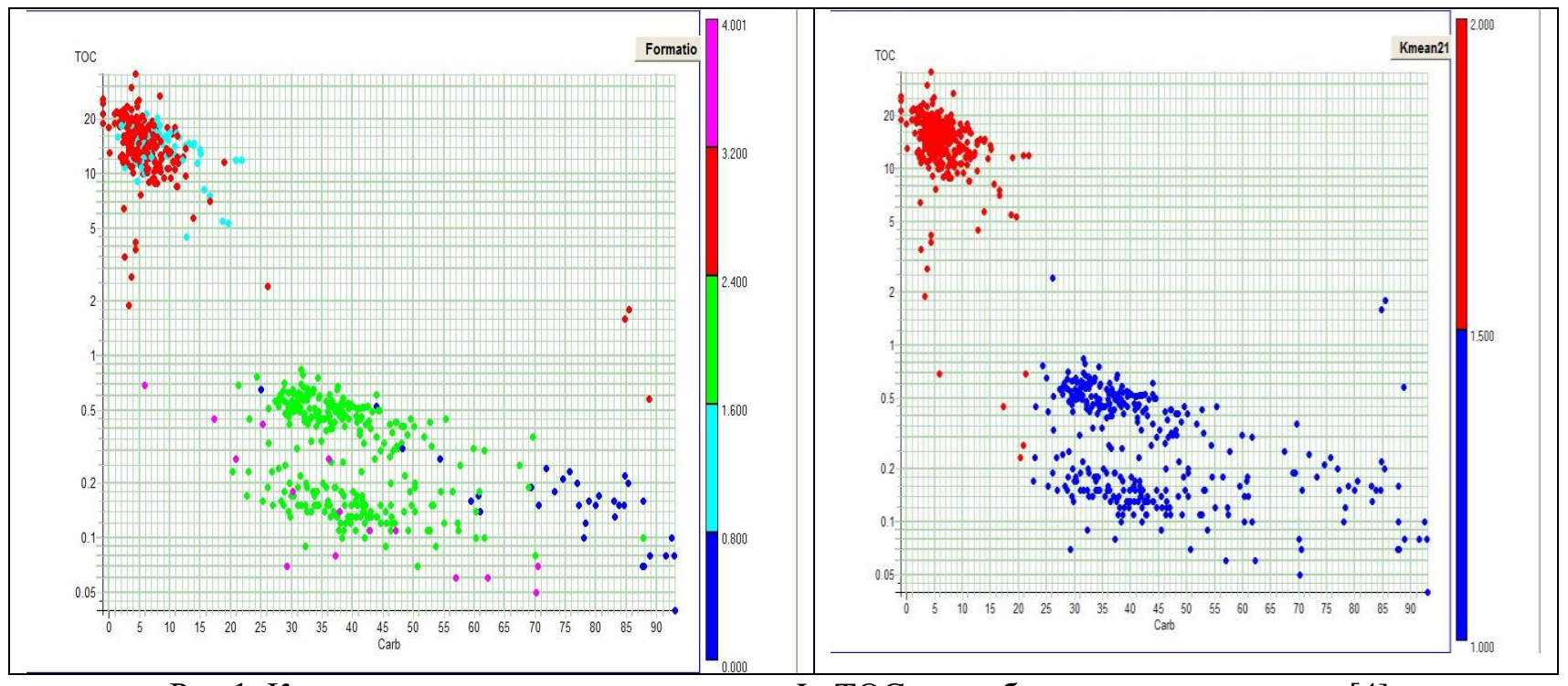

Рис 1. Корреляционное поле взаимосвязи LgTOC и карбонатности по данным [4]. Слева цветокодом указаны отложения Scallion, U.Bakken, M. Member, L.Bakken, Three Forks. Справа цветом дифференцировано расчленение на два кластера алгоритмом kMean.,

В массиве Cline существенно меньше образцов и существенно больше измерений. Характер взаимосвязи компонент при этом достаточно диффузный, за исключением нескольких парных связй (ТОС и плотности; плотности и карбонатности, ТОС и содержания пирита, глинистости и содержания полевых шпатов) 


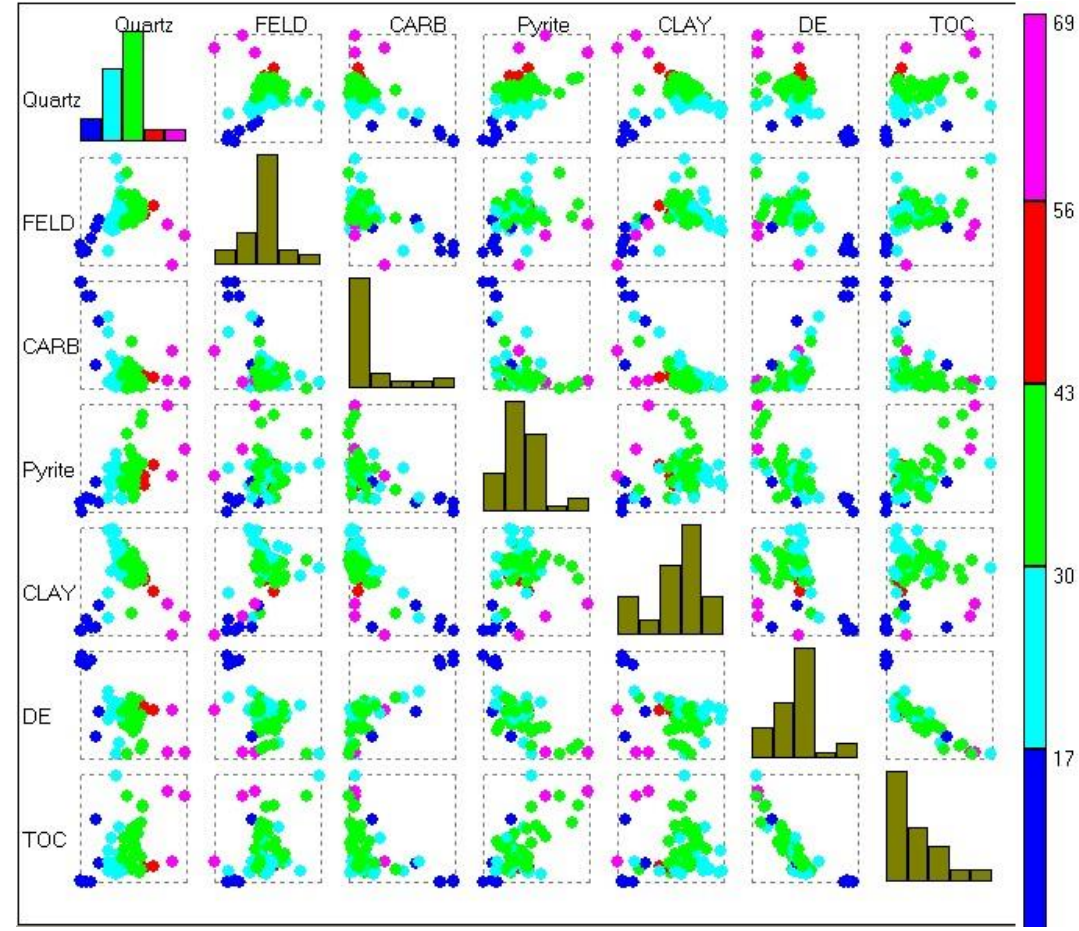

Рис 2 Матричный плот содержания базовых компонент и плотности по массиву Cline (цветокодирование по содержанию кварца)

Попытки построения регрессии на ТОС методом Эфроимсона производились с участием и без участия плотности. В случае включения в состав регрессии плотности она выбиралась как первый регрессор с коэффициентом детерминации равным 0.868 и включение дальнейших регрессоров повышало коэффициент детерминации только до $0.899 . \quad$ В случае исключения плотности получался более привычный на глаз петрофизика результат, приведенный на Рис 3.
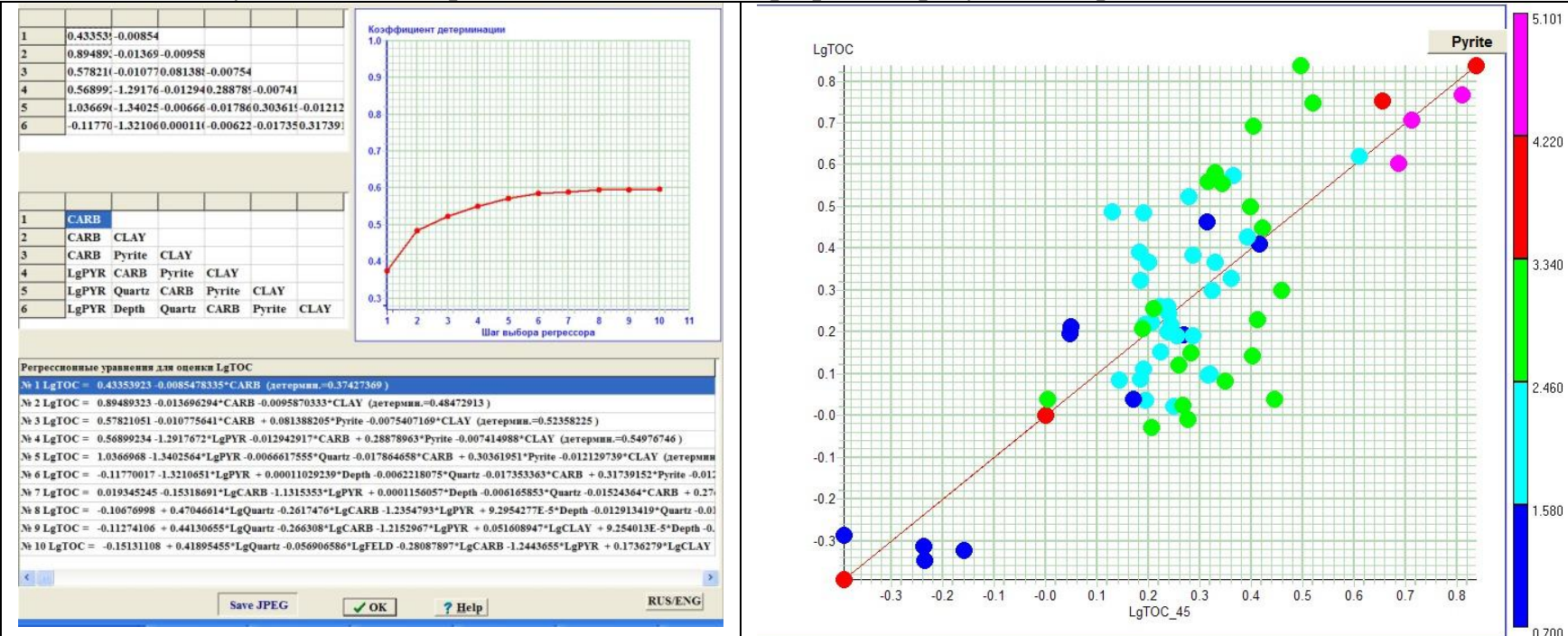

Рис 3. Слева кривая роста коэффициента детерминации (квадрат коэффициента корреляции) по мере наращивания регрессоров. Справа полученное по регрессии сопоставление прогнозного значения ТОС с измеренным (цветокодирование по содержанию пирита)

Кривая роста показывает, что на первом шаге в качестве регрессора выбирается суммарная карбонатность, на втором - глинистость, на третьем - содержание пирита и только на пятом добавляется в качестве регрессора кварц, а на шестом глубина.

Вызывают интерес как минимум два вопроса. Первый из них вопрос о генезисе отложений и о природе этой взаимосвязи и второй об уровне её устойчивости. Ответ на первый вопрос уместно искать, используя данные химического анализа и индикаторы типа окислительно- 
восстановительной обстановки. Обработка доступных массивов с данными о химическом составе показывает преобладающее влияние содержания таких элементов как ванадий, никель и молибден (традиционно связываемых с восстановительной средой осадконакопления). Для ответа на второй вопрос желательно привлечение дополнительных данных по другим объектам. В качестве таких массивов был выбран композитный массив, охватывающий данные по сланцевым резервуарам Китая. Часть полученных результатов приведена на Рис 4
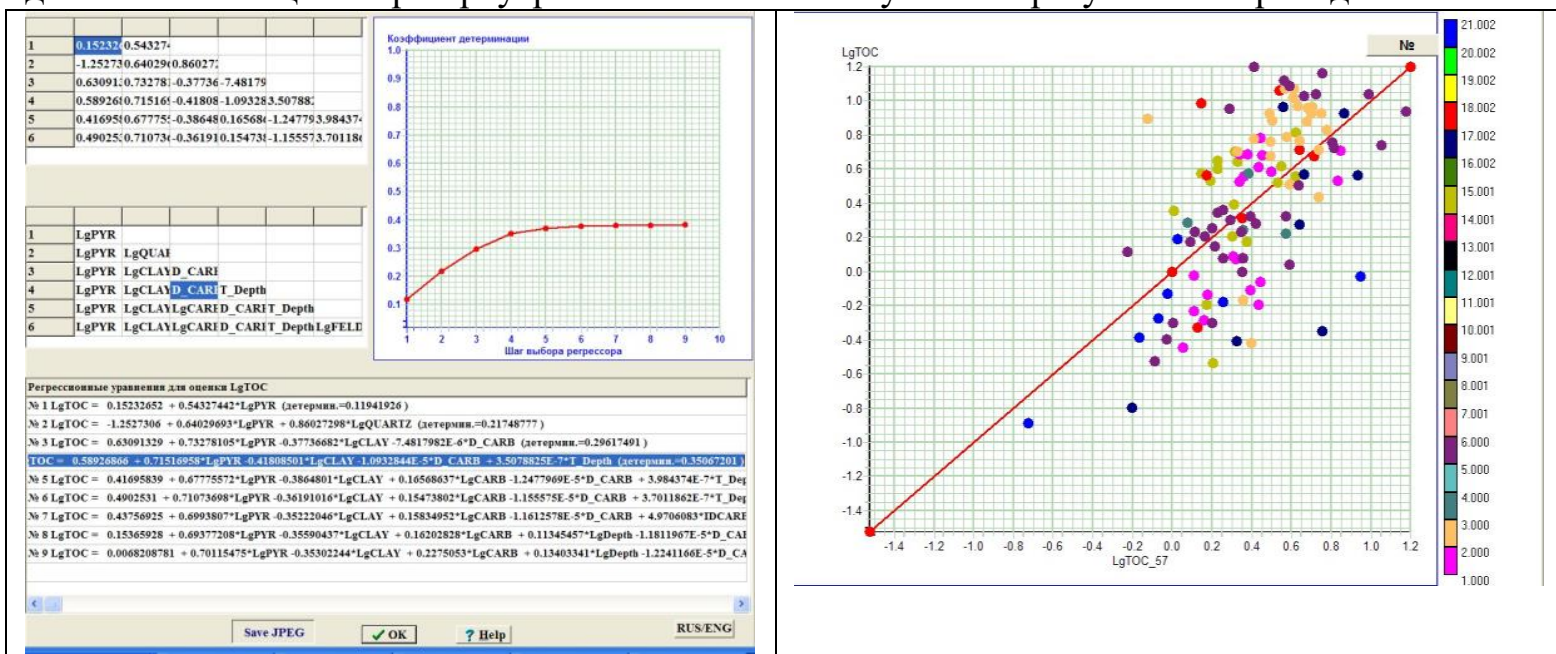

Рис 4. Слева кривая роста коэффициента детерминации (квадрат коэффициента корреляции) по мере наращивания регрессоров. Справа полученное по регрессии сопоставление прогнозного значения ТОС с измеренным (цветокодирование по номеру включенной в выборку коллекции образцов)

\section{Выводы}

1. На эмпирических данных показана возможность предсказания ТОС методами регрессионного и кластерного анализа в условиях конкретных сланцевых отложений 2. Показана существенная роль в таких прогнозах пирита (для отложений с высоким ТОС)

\section{Литература}

1. Еникеев Б.Н. О современном состоянии петрофизического обоснования интерпретации каротажа и керна, ориентированном на использование априорных знаний//Пути реализации нефтегазового и рудного потенциала ХМАО - Югры / Материалы 17 научной конференции.-Том. 1.-Ханты-Мансийск.-2014.-С. 294-304. https://independent.academia.edu/BorisEnikeev

2. Еникеев Б.Н. Опыт сравнительного изучения и обоснования петрофизических взаимосвязей Пути реализации нефтегазового и рудного потенциала ХантыМансийского Автономного Округа - ЮГРЫ'Восемнадцатая научная конференция том. 1 Ханты-Мансийск ОАО " Тюменский дом печати " 2015 стр. 180-193 https://independent.academia.edu/BorisEnikeev

3. Еникеев Б.Н., Колесов В.В. Некоторые особенности петрофизической модели коллекторов баженовской свиты. EAGE/SPE Joint Workshop 2017. Shale Science: Prospecting \& Development 10-11 April 2017, Moscow, Russia

4. Daniel M. Jarvie, Robert J. Coskey, Michael s. Johnson, Jay E. Leonard Platte. The Geology and Geochemistry of the Parshall Area, Mountrail County, North Dakota Revisiting and Revitalizing the Niobrara in the Central Rockies, J.E. Estes-Jackson, D.S. Anderson, eds. Denver, Colo.: Rocky Mountain Association of Geologists, 2011.

5. Lauren Patricia Redmond Lithofacies, Depositional Systems, and Depositional Model of the Mississippian Barnett Formation in the Southern Fort Worth Basin Thesis The University of Texas at Austin 2016 


\section{References}

1. Enikeev B.N. On the current state of the petrophysical substantiation of the interpretation of the $\log$ and core, focused on the use of a priori knowledge/ Khanty-Mansiysk Scientific Conference XVII Materials -Vol. 1.- Khanty-Mansiysk.-2014.-pp. 294-304. https://independent.academia.edu/BorisEnikeev

2. Enikeev B.N. Experience in comparative study and substantiation of petrophysical relationships // Khanty-Mansiysk Scientific Conference XVIII Materials -Vol. 1.- KhantyMansiysk.- 2015 pp. 180-193 https://independent.academia.edu/BorisEnikeev

3. Enikeev B.N., Kolesov V.V. Some features of the petrophysical model of the Bazhenov suite EAGE/SPE Joint Workshop 2017. Shale Science: Prospecting \& Development 10-11 April 2017, Moscow, Russia

4. Daniel M. Jarvie, Robert J. Coskey, Michael s. Johnson, Jay E. Leonard Platte. The Geology and Geochemistry of the Parshall Area, Mountrail County, North Dakota Revisiting and Revitalizing the Niobrara in the Central Rockies, J.E. Estes-Jackson, D.S. Anderson, eds. Denver, Colorado.: Rocky Mountain Association of Geologists, 2011.

5. Lauren Patricia Redmond Lithofacies, Depositional Systems, and Depositional Model of the Mississippian Barnett Formation in the Southern Fort Worth Basin Thesis The University of Texas at Austin 2016 